\title{
ENVEJECIMIENTO Y CALIDAD SEMINAL: UN ANÁLISIS DE 9.168 CASOS EN CÓRDOBA, ARGENTINA
}

\author{
Rosa Isabel Molina', Ana Carolina Martini², Andrea Tissera', José Olmedo', Daniel Senestrari', \\ Marta Fiol de Cuneo², y Rubén Daniel Ruiz².
}

'Laboratorio de Andrología y Reproducción (LAR). Córdoba. Argentina.

2Investigadores del Consejo Nacional de Investigaciones Científicas y Tecnológicas (CONICET). Instituto de Fisiología. Facultad de Ciencias Médicas. Universidad Nacional de Córdoba. Córdoba. Argentina.

\begin{abstract}
Resumen.- OBJETIVO: En coincidencia con la actual tendencia hacia la paternidad tardía, son necesarios más estudios para establecer la relación entre la edad del varón y las características seminales.

El objetivo del presente trabajo fue evaluar la relación de la edad con la calidad espermática y con los niveles seminales de marcadores funcionales del epidídimo y de glándulas anexas.
\end{abstract}

MÉTODOS: este estudio retrospectivo fue realizado sobre 9168 casos obtenidos de hombres miembros de parejas con problemas de fertilidad que se presentaron en el Laboratorio de Andrología y Reproducción, en Córdoba, Argentina durante 10 años (1995-2004)

\section{CORRESPONDENCIA}

Ana Carolina Martini

Instituto de Fisiología

Facultad de Ciencias Médicas

Universidad Nacional de Córdoba

Santa Rosa 1085

X5000ESU Córdoba. (Argentina)

acmartini2000@yahoo.com

Aceptado para publicar: 24 de noviembre de 2009 (en pacientes entre 20 y 77 años). Se controló estadísticamente un importante número de posibles factores de confusión tales como tiempo de abstinencia sexual, hábitos tóxicos, condiciones laborales y consumo de medicamentos. Los parámetros evaluados fueron: volumen seminal, concentración espermática, número total de espermatozoides, motilidad, morfología y vitalidad espermática. Se cuantificaron además las concentraciones seminales de alfa-glucosidasa, fructosa y ácido cítrico.

RESULTADOS: en el presente estudio detectamos un descenso significativo en el volumen seminal, el recuento espermático, la motilidad, la vitalidad y el porcentaje de espermatozoides morfológicamente normales, asi como una reducción en los niveles de alfa-glucosidasa y fructosa seminales en relación con la edad.

CONCLUSIONES: dado que la calidad seminal constituye un factor de pronóstico para la fertilidad masculina, las evidencias indican que los hombres podrían disminuir su fertilidad a medida que envejecen. Aquellas parejas que decidan postergar su paternidad deben ser advertidas acerca de este hecho.

Palabras clave: Envejecimiento. Calidad seminal. Volumen seminal. Recuento espermático. Motilidad. Vitalidad. Morfología espermática. Alfaglucosidasa. Fructosa. Ácido cítrico.

Summary.- OBJECTIVES: Concomitantly with the actual trend towards later fathering, more detailed studies are necessary to establish the relationship between male age and seminal features.

The objective of the present paper was to evaluate the relationship of men age with semen quality and with 
the seminal levels of epididymal and accessory gland markers.

METHODS: The study was conducted as a retrospective study of 9168 cases obtained from the Andrology and Reproduction Laboratory in Cordoba, Argentina for 10 years (1995-2004) (men ages 20 to 77). An important number of factors such as abstinence time, toxic habits, work conditions and drugs consumption has been statistically considered. The parameters measured were: seminal volume, sperm concentration, total sperm count, sperm motility, morphology and viability. Seminal levels of alpha-glucosidase, fructose and citric acid were also evaluated.

RESULTS: We detected a significant decrease in seminal volume, sperm count, motility, viability and normal morphology, and a reduction in alpha-glucosidase and fructose levels in relation to age.

CONCLUSIONS: Since semen quality is a tool for fertility prognosis estimation, the weight of evidence indicates that men may become progressively less fertile as they get older. Couples who decide to delay childbearing should be warned about this matter.

Keywords: Aging. Sperm quality. Semen volume. Sperm count. Motility. Viability. Sperm morphology. Alpha-glucosidase. Fructose. Citric acid.

\section{INTRODUCCIÓN}

A diferencia de las mujeres, los hombres en general pueden concebir hijos más allá de sus 40 años. No se le reconoce a la función reproductiva masculina un cese abrupto y la espermatogénesis continúa sin que exista un límite conocido $(1,2)$. De hecho, más allá de que es esperable que los niveles de andrógenos plasmáticos disminuyan concomitantemente con la edad, en la mayoría de los individuos permanecen dentro del rango normal (3). Sin embargo, algunos estudios revelan que un retraso en la paternidad puede disminuir las probabilidades de concepción, no solamente en parejas que consultan por infertilidad sino también en las fértiles (4-6).

El análisis de la bibliografía relacionada sugiere que el aumento en la edad del hombre está asociada a una disminución en el volumen seminal, en la motilidad espermática y en el porcentaje de espermatozoides morfológicamente normales, sin que exista consenso en lo referente a concentración espermática $(1,2,7)$.

A pesar de que los parámetros mencionados pueden sufrir modificaciones por la función alterada del epidídimo y/o de las glándulas accesorias, sólo un pequeño número de estudios analizan los niveles seminales de marcadores tales como alfa-glucosidasa, fructosa, zinc y ácido cítrico, como evidencia del deterioro en la función de estas estructuras.

Asimismo, si bien la influencia de sustancias tóxicas tales como alcohol, tabaco, pesticidas, drogas, etc es bien conocida (8-13), sólo en unos pocos estudios se controlan estadísticamente estos factores con el fin de evitar la malinterpretación de los resultados.

Teniendo en cuenta la tendencia actual a retrasar la paternidad, Kidd y col (2) declararon que son necesarios estudios más detallados con el fin de establecer la relación entre la edad del hombre y las características seminales, por ejemplo, incluyendo un número adecuada de voluntarios en todo el espectro etario, controlando posibles factores de confusión y seleccionando grupos comparativamente adecuados.

En base a lo expuesto previamente, los objetivos del presente trabajo fueron evaluar, en un importante número de pacientes estudiados en un laboratorio de andrología en Córdoba (Argentina) durante 10 años, la relación de la edad con la calidad seminal y con los niveles seminales de marcadores de la función epididimaria, de las vesículas seminales y de la próstata. Para evitar la interpretación errónea de los resultados, se controlaron estadísticamente un número importante de factores tales como días de abstinencia, hábitos tóxicos, condiciones laborales y consumo de medicamentos.

\section{MATERIAL Y MÉTODOS}

Las muestras de semen fueron obtenidas de pacientes, miembros de parejas con problemas de fertilidad, que asistían a un laboratorio de andrología (Laboratorio de Andrología y Reproducción -LAR-) en Córdoba, Argentina. Fueron analizados datos obtenidos durante 10 años (1995 a 2004). Los pacientes llenaron voluntariamente un formulario conteniendo información sobre hábitos tóxicos, consumo de drogas y enfermedades previas. Se excluyeron de este estudio, formularios incompletos o aquellos pacientes que informaban abstinencia sexual fuera del rango (2-10 días), métodos de recolección de la muestra diferentes a la masturbación y/o inconvenientes durante el proceso de recolección. Finalmente, se consideraron para este estudio 9168 muestras de semen (1/paciente).

\section{Parámetros seminales evaluados:}

Luego de la licuefacción, el análisis del semen se realizó de acuerdo a las recomemndaciones 
de la Organización Mundial de la Salud (OMS) (14).

El voluman seminal se determinó en un tubo cónico graduado. La concentración y motilidad espermática se contabilizaron en cámara de Makler según método convencional (Sefi-Medical Instrument,
Haifa, Israel) (15). Los resultados se informaron como porcentaje de espermatozoides móviles $(A+B)$ (espermatozoides rápidos + lentos). En el grupo de células inmóviles se evaluó mediante tinción suprevital de eosina la población de espermatozoides viables (16) y los resultados se expresaron como porcentaje de espermatozoides muertos. La sumatoria del porcentaje

\section{TABLA I. CARACTERÍSTICAS GENERALES DE LA POBLACIÓN INCLUIDA EN ESTE ESTUDIO: PACIENTES QUE CONCURRÍAN A UN LABORATORIO DE ANDROLOGÍA IN CÓRDOBA, ARGENTINA.}

\begin{tabular}{|c|c|c|}
\hline & Características & $\%$ de los pacientes \\
\hline Año del análisis seminal & $\begin{array}{l}1995-1996 \\
1997-1998 \\
1999-2000 \\
2001-2002 \\
2003-2004\end{array}$ & $\begin{array}{l}12.6 \\
19.6 \\
21.1 \\
20.3 \\
26.5\end{array}$ \\
\hline Abstinencia (días) & $\begin{array}{c}2 \\
3-5 \\
6-10\end{array}$ & $\begin{array}{l}14.4 \\
68.8 \\
16.8\end{array}$ \\
\hline Patología urogenital & $\begin{array}{c}\text { Varicocele } \\
\text { Criptorquidia } \\
\text { Enfermedad inflamatorio o infecciosa } \\
\text { Cirugía urogenital } \\
\text { Otras }\end{array}$ & $\begin{array}{l}22.0 \\
1.5 \\
2.4 \\
11.5 \\
4.5\end{array}$ \\
\hline Exposición a tóxicos & $\begin{array}{l}\text { Tabaco } \\
\text { Alcohol } \\
\text { Otros }\end{array}$ & $\begin{array}{l}15.5 \\
13.4 \\
1.3\end{array}$ \\
\hline Medicación & $\begin{array}{l}\text { Antiinflamatorios no esteroideos } \\
\qquad \begin{array}{c}\text { Hormonas } \\
\text { Antibióticos } \\
\text { Pentoxifilina } \\
\text { Otros }\end{array}\end{array}$ & $\begin{array}{l}8.6 \\
0.9 \\
0.3 \\
0.3 \\
2.8\end{array}$ \\
\hline
\end{tabular}


de espermatozoides rápidos, lentos, viables inmóviles y muertos comprenden el 100\% de las gametas. La morfología espermática fue evaluada empleando la tinción de Papanicolaou y de acuerdo a los criterios de la OMS (14).

\section{Análisis estadístico:}

El contraste de los valores seminales entre los grupos etarios fue realizado mediante el método inverso de Helmert en un análisis de varianza (ANOVA), describiendo gráficamente los resultados para cada grupo etario como media y su correspondiente intervalo de confianza del $95 \%$. En este test, la media de cada grupo etario se compara con la media combinada de todos los grupos anteriores (excepto el primero). Cuando fue necesario, la escala natural de algunos parámetros se transformó a una distribución gaussiana mediante raíz cuadrada o función logarítmica.

El análisis previo fue repetido incluyendo en el modelo cada uno de los factores de confusión como covariable (ANCOVA). En todos los casos, el sesgo resultó nulo o levemente significativo. Los factores de confusión considerados fueron: días de abstinencia, año en que se realizó el análisis, exposición ambiental y/o tóxica (tabaco, alcohol, pesticidas, metales pesados, radiación y otros), patologías urogenitales (tales como varicocele, criptorquidia, enfermedades inflamatorias o infecciosas, cirigía urogenital y otros) y medicación actual (particularmente antiinflamatorios no esteroideos, hormonas, antibióticos, pentoxifilina, drogas prescriptas para el tratamiento de enfer-

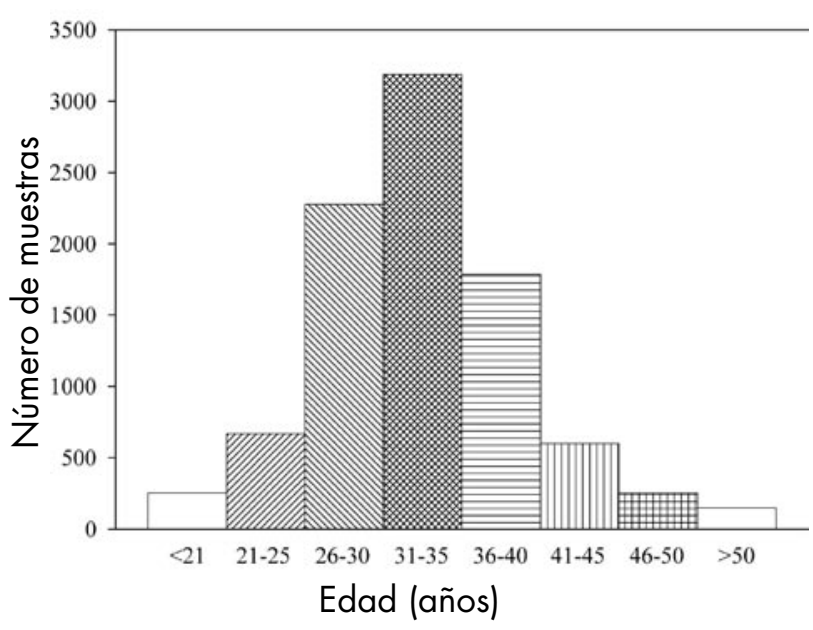

FIGURA 1. Número de muestras evaluadas

(1 muestra/paciente) en cada grupo etario de pacientes atendidos en un laboratorio de andrología en Córdoba, Argentina. medades cardiovasculares o diabetes, psicotrópicos y otros).

Para considerar significación estadística y rechazar la hipótesis nula aplicamos un valor de alfa menor al $5 \%(p<0.05)$. En todos los casos, $n$ representa el número de muestras de semen evaluadas. Todos los análisis estadísticos fueron realizados con SPSS $尺 11.5$.

\section{RESULTADOS}

En la Tabla I se muestran las caraterísticas generales de la población incluida en el presente estudio. Las 9168 muestras de semen evaluadas en el presente estudio fueron agrupadas en intervalos etarios tal como se representa en la Figura 1. El mayor número de muestras se obtuvo para los pacientes de 31 a 35 años, con la mayoría de los casos incluída entre los 21 y 45 años de edad.

Como puede observarse en la Figura 2, la calidad seminal disminuyó con la edad. El volumen seminal dsiminuyó significativamente en pacientes con más de 50 años y fue paralelo a una disminución estadísticamente significativa en el número total de espermatozoides.

La motilidad espermática (rápidos + lentos) así como la vitalidad (expresada como \% de espermatozoides muertos) disminuyeron con la edad, ambas en forma significativa desde la segunda mitad de la tercera década. Ya que los pacientes que exhibieron concentraciones espermáticas menores a 0.15 millones no fueron considerados, el número de muestras evaluadas en el parámetro anteriormente descripto fue de 8613 .

El porcentaje de espermatozoides morfológicamente normales de acuerdo a los criterios de la OMS disminuyó significativamente en pacinetes con más de 50 años de edad. La evaluación de la morfología espermática de acuerdo al criterio estricto de Kruger mostró una tendencia similar (resultados no mostrados).

Los resultados obtenidos de la evaluación bioquímica de las muestras de semen se presentan en la Figura 3. No se detectaron diferencias en los niveles de alfa-glucosidasa en las muestras ente 20 y 49 años de edad $162.45 \mathrm{mU} /$ eyaculado; IC95\% 60.9-64.0). Desde 50 a 59 años, la concentración seminal de alfa-glucosidasa alcanzó una media de $47.34 \mathrm{mU} /$ eyaculado (IC95\% 39.9-55.5) y disminuyó significativamente con respecto a la media obtenida del intervalo 20-49 años de edad ( $p<0.01)$. 

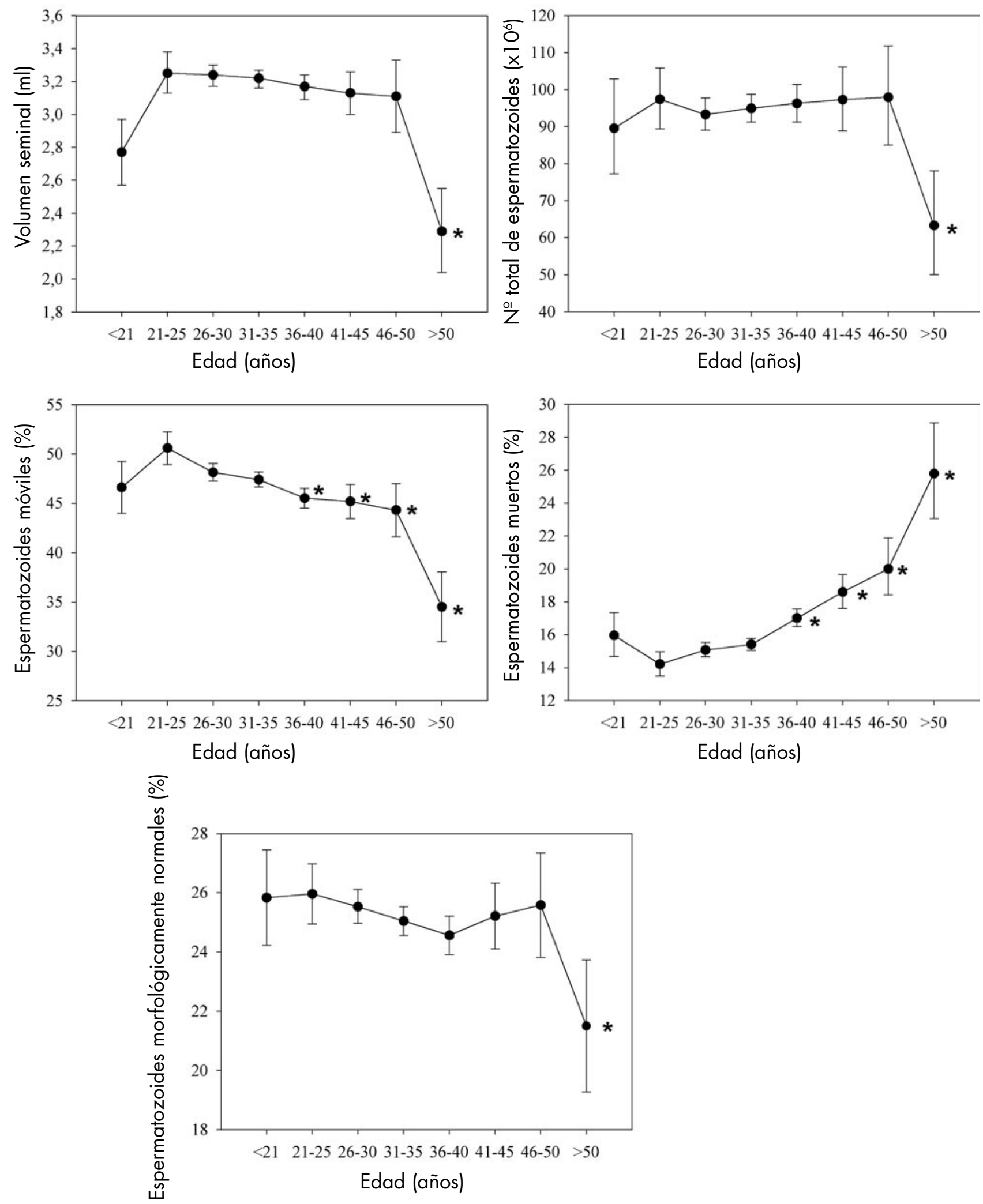

FIGURA 2. Parámetros seminales de pacientes atendidos en un laboratorio de andrología en Córdoba, Argentina, agrupados en diferentes intervalos etarios. La morfología espermática fue cuantificada de acuerdo a las recomendaciones de la OMS. Para la evaluación de motilidad y vitalidad, no se consideraron las muestras de semen con concentraciones inferiores a $0.15 \times 10^{6} / \mathrm{ml}$. El número de muestras evaluadas fue: 9.168 para volumen seminal y recuento espermático, 7.167 para morfología espermática y 8.613 para motilidad y vitalidad. *: p< 0.001 o

0.05 (para motilidad espermática) por contraste inverso de Helmert. 

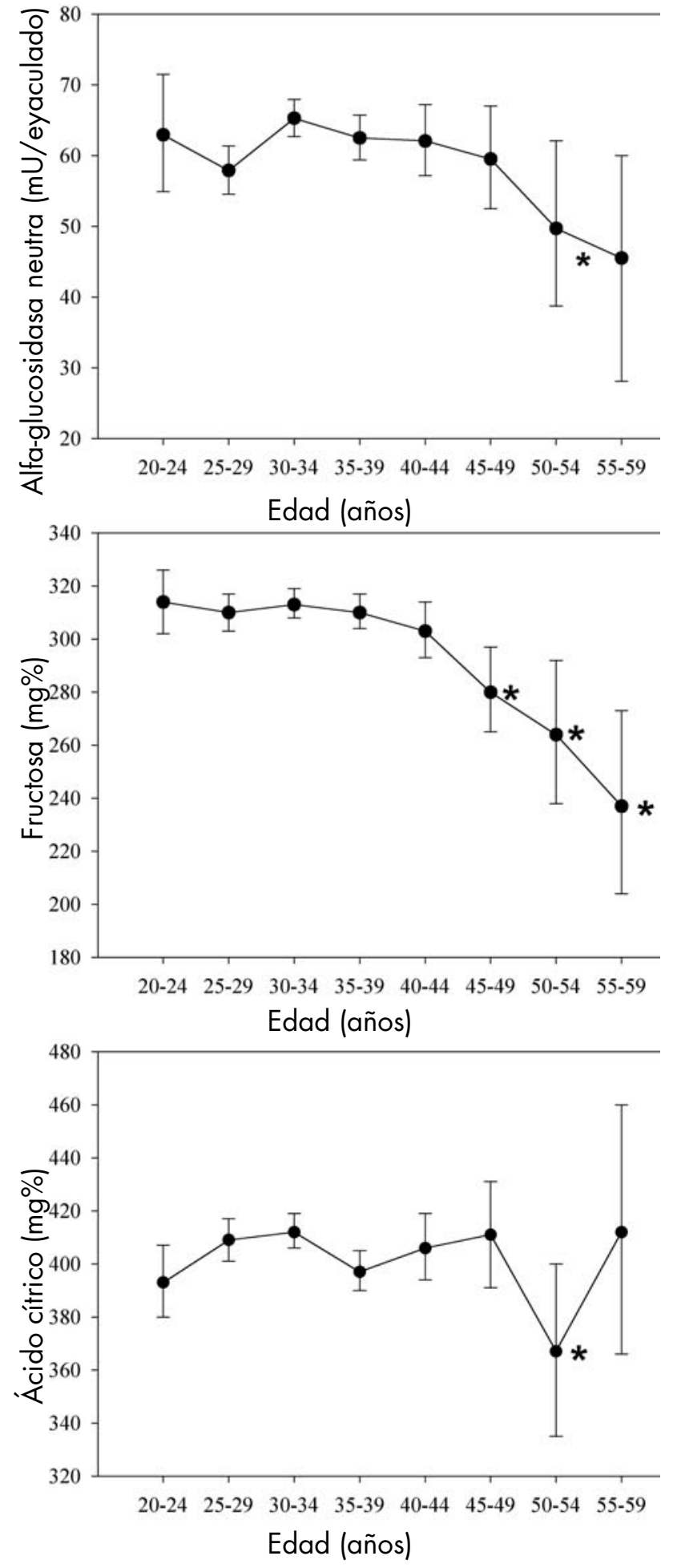

FIGURA 3. Parámetros bioquímicos del semen de pacientes atendidos en un laboratorio de andrología en Córdoba, Argentina, agrupados en diferentes rangos etarios. El número de muestras evaluadas fue: 2618 para alfa-glucosidasa neutra, 7211 para fructosa y 8981 para ácido cítrico. ${ }^{*}: p<0.01$ por contraste inverso de Helmert. Los valores medios para el intervalo 50-59 fueron significativamente menores que los del intervalo 20-49 ( $p<0.011$.
Los valores seminales de fructosa también disminuyeron con la edad; desde los 45 años los resultados alcanzaron significación estadística, con una disminución lineal de 4.5 unidades/año $(r=$ $0.131 ; p<0.05)$.

Exceptuando por un valor medio inusualmente bajo en los niveles de ácido cítrico en el rango de 50-54 años, no se obtuvieron diferencias significativas en este parámetro con relación a la edad. Tampoco se detectaron diferencias significativas en el tiempo de licuefacción (resultados no mostrados).

\section{DISCUSIÓN}

Eskenazi y col (17) sugirieron que las alteraciones en los parámetros seminales en relación a la edad podrían ser producidas al menos de dos formas diferentes.

En primer lugar, tal como ha sido demostrado para la fertilidad femenina, podrían ocurrir cambios celulares o fisiológicos en el tracto genitourinario provocados probablemente por la edad per se y/o por posibles mecanismos genéticos, por ejemplo cambios morfológicos en el testículo envejecido $(6,18,19)$, disminución en la función epididimaria, de las vesículas seminales y/o la próstata $(17,20)$, cambios en los niveles de testosterona y hormonas relacionadas (3) y disminución en la capacidad de reparación del daño celular o tisular.

En segundo lugar, la edad aumenta la probabilidad de sufrir daño reproductivo por factores tales como infecciones urogenitales, enfermedad vascular y/o acumulación de sustancias tóxicas tales como alcohol, tabaco, pesticidas, etc $(1,4,17)$. debido a:

Los datos del presente trabajo son robustos

a) el gran número de hombres evaluados;

b) la corrección estadística de un gran número de factores de confusión que fue posible gracias al gran número de muestras;

c) el amplio rango de edad considerado;

d) la evaluación de marcadores seminales; esta estimación provee información valiosa acerca de la calidad seminal y no ha sido considerada en la mayor parte de los estudios previos;

e) el hecho de que Córdoba es un área metropolitana rodeada de comunidades rurales, que reúne perso- 
nas con diferentes estilos de vida y/o hábitos y

f) el hecho de que los resultados del presente estudio han sido obtenidos en un solo laboratorio y que los análisis han sido realizados por dos técnicos bien entrenados; de esta forma se evitan las diferencias metodológicas y experimentales entre los distintos laboratorios (una característica inevitable de los estudios multicéntricos).

En concordancia con la mayor parte de los trabajos publicados $(2,7,17,21-27)$, en el presente estudio detectamos que el envejecimiento se caracteriza por una disminución estadísticamente significativa en el volumen seminal, el recuento espermático, la motilidad y la vitalidad y con un aumento en el porcentaje de espermatozoides morfológicamente anormales. Cabe destacar, que la calidad seminal de los pacientes menores de 21 años tendió a disminuir (aunque no significativa) con respecto a 21-50 años. Este fenómeno merece ser investigado.

Con respecto al volumen seminal, los factores determinantes conocidos de mayor importancia sobre este parámetro son el tiempo desde la última eyaculación (28) y la secreción de las vesículas seminales, que proveen el $70 \%$ del plasma seminal $(20$, 29). En el presente estudio, el tiempo de abstinencia fue controlado estadísticamente y esta variable aumentó, poco pero en forma significativa, con la edad. Teniendo en cuenta la disminución en la frecuencia sexual con la edad, $\mathrm{Ng}$ y col (7) sugirieron que la disminución en el volumen seminal se explicaría más por una función androgénica deficiente, patologías subclínicas de las glándulas accesorias y/o defectos en la eyaculación acumulados con la edad más que a una reducción en el período de abstinencia. En concordancia con este postulado, cuando se evaluaron los niveles de fructosa seminales (marcador funcional de las vesículas seminales), se detectó una disminución progresiva y estadísticamente significativa en pacientes mayores de 45 años.

Considerando que el volumen seminal disminuye con al edad, el hecho de que diversos autores no hayan encontrado disminución en la concentración espermática no es sorprendente $(7,17)$ y no prueba que la espermatogénesis haya permanecido sin cambios. En este contexto, debemos resaltar la disminución de la cantidad total de espermatozoides, que ha sido efectivamente demostrada por nosotros y por otros autores $(7,23)$. Asimismo, ya que la morfología espermática constituye otro parámetro que refleja el estado del epitelio germinal $(30,31$, 32), la disminución simultánea en el porcentaje de espermatozoides morfológicamente normales detectada en nuestro estudio, sugiere la ocurrencia de cierto nivel de alteración en el proceso espermatogénico.

Con respecto a la motilidad espermática, detectamos una disminución estadísticamente significativa en este parámetro desde los 35 años de edad, paralela a un aumento en el porcentaje de espermatozoides muertos. La pendiente de la curva de disminución de la motilidad es mayor en el último intervalo etario $(\geq 46)$ y se acompañó de niveles seminales menores de alfa-glucosidasa neutra, marcador de la función epididimaria, estructura en la cual los espermatozoides adquieren motilidad (33). En concordancia con nuestros resultados, Henkel y col (34) encontraron que tanto en pacientes andrológicos como en donantes de semen, la edad se correlacionaba negativamente con la motilidad espermática y con los niveles de testosterona plasmática y positivamente con el porcentaje de flagelos anormales y de zinc flagelar; ambos parámetros reflajan la maduración epididimaria. Estos autores postularon, que puesto que el epidídimo es una estructura funcionalmente dependiente de testosterona, la maduración epididimaria podría estar alterada durante el envejecimiento masculino. Sin embargo, Pasqualotto y col (23) informaron de una disminución en la motilidad espermática concomitante con la edad que no se acompañaba de modificaciones en los niveles de testosterona, sugiriendo que la deficiencia de andrógenos no es la única causa de la reducción en la motilidad. Ya que el presente es un estudio retrospectivo, no podemos evaluar este parámetro.

Ha sido publicado que el tiempo de licuefacción, parámetro que depende de las secreciones prostáticas (35), se encontraba prolongado en sujetos mayores con respecto a los más jóvenes y sugirieron que estos cambios podrían disminuir la motilidad espermática. En el presente estudio no encontramos alteraciones en relación a la edad ni en el tiempo de licuefacción ni en los niveles seminales de citrato (excepto por un valor inusualmente bajo el el rango etario 50-54).

\section{CONCLUSIÓN}

Siendo la calidad seminal una herramienta predictiva de la fertilidad, el peso de las evidencias indica que los hombres pueden volverse progresivamente menos fértiles a medida que envejecen. En su review, Kühnert \& Nieschlag (1) concluyeron que a pesar de que los efectos del envejecimiento masculino son menos prominentes que los femeninos, los hombres comienzan a contribuir con la disminución de la fertilidad en una pareja a finales de la tercera década y con la disminución de la fecundidad a 
principios de la cuarta década. Las tendencias detectadas en nuestro estudio coinciden con esta observación. Aquellas parejas que decidan retrasar su paternidad deberían ser advertidas de este hecho.

\section{AGRADECIMIENTOS}

Este trabajo fue realizado con subsidios de la Secretaría de Ciencia y Técnica (SECyT), Universidad Nacional de Córdoba.

\section{BIBLIOGRAFIA y LECTURAS RECOMENDADAS (*lectura de interés $y^{* *}$ lectura fundamental)}

**1. Kühnert B, Nieschlag E. Reproductive functions of the ageing male. Hum. Reprod. Up, 2004; 10: 327.

**2. Kidd SA, Eskenazi B, Wyrobek AJ. Effects of male age on semen quality and fertility: a review of the literature. Fertil. Steril, 2001; 75: 237.

**3. World Health Organization Men. Aging, and Health: Achieving Health Across the Life Span. World Health Organization, Geneva, 2001.

4. La Rochebrochard E, Thonneau P. Paternal age $\geq 40$ years: an important risk factor for infertility. Am. J. Obstet. Gynecol, 2003; 189: 901.

5. Dunson DB, Colombo B, Baird DD. Changes with age in the level and duration of fertility in the menstrual cycle. Hum. Reprod, 2002; 17: 1399.

6. Ford WCK, North K, Taylor H, Farrow A, Hull MGR, Golding J, et al. Increasing paternal age is associated with delayed conception in a large population of fertile couples: evidence for declining fecundity in older men. Hum. Reprod3, 2000; 15: 170.

**7. Ng KK, Donat R, Chan L, Lalak A, Di Piero I, Handelsman DJ. Sperm output of older men. Hum. Reprod, 2004; 19: 1811.

8. Martini AC, Molina R, Estofán D, Senestrari D, Fiol de Cuneo M, Ruiz RD. Effects of alcohol and cigarette consumption upon human seminal quality. Fertil. Steril, 2004; 82: 374.

9. Martini AC, Molina RI, Tissera AD, Ruiz RD, Fiol de Cuneo M. Analysis of semen from patients chronically treated with low or moderate doses of aspirin-like drugs. Fertil. Steril, 2003; 80: 221.

10. Pflieger-Bruss S, Schuppe HC, Schill WB. The male reproductive system and its susceptibility to endocrine disrupting chemicals. Androl, 2004 36: 337.

11. Queiroz EK, Waissmann W. Occupational exposure and effects on the male reproductive system. Cad. Saude Púb, 2006; 22: 485.
12. Kumar S. Occupational exposure associated with reproductive dysfunction. J. Occup. Health, 2004; 4: 1 .

13. Bouvet BR, Paparella CV, Feldman RN. Efecto del tabaquismo sobre la espermatogénesis en hombres con infertilidad idiopática. Arch. Esp. Urol, 2007; 60: 273.

**14. World Health Organization. WHO laboratory manual for the examination of human semen and sperm-cervical mucus interaction. Cambridge University Press, Cambridge, 1992.

15. Makler A. The improved ten microliter chamber for rapid sperm count and motility evaluation. Fertil. Steril, 1980; 33: 337.

16. Eliasson R. Supravital staining of human spermatozoa. Fertil. Steril, 1977; 28: 1257.

**17. Eskenazi B, Wyrobek AJ, Sloter E, Kidd SA, Moore L, Young S, et al. The association of age and semen quality in healthy men. Hum. Reprod, 2003; 18: 447.

*18. Bishop MW. Aging and reproduction in the male. J. Reprod. Fertil, 1970; 12: 65.

19. Auger J, Jouannet P. Age and male fertility: biological factors. Rev. épidém. san. publ, 2005; 53: 2825.

20. Goldman N, Montgomery M. Fecundability and husband's age. Social Biol, 1989; 36: 146.

*21. Rolf C, Behre HM, Nieschlag E. Reproductive parameters of older compared to younger men of infertile couples. Int. J. Androl, 1996; 19, 135.

*22. Hellstrom WJ, Overstreet JW, Sikka SC, Denne J, Ahuja S, Hoover AM, et al. Semen and sperm reference ranges for men 45 years of age and older. J. Androl, 2006; 27: 421.

*23. Pasqualotto FF, Sobreiro BP, Hallak J, Pasqualotto EB, Lucon AM. Sperm concentration and normal sperm morphology decrease and follicle-stimulating hormone level increases with age. BJU Int, 2005; 96: 1087.

24. Cánovas-García JA, Cadenas-García VP, MolinaGasset R, Fernández-García JS, Sánchez-DíazA. F, García-Zamora J.A. Relación entre la edad del varón y la calidad del estudio seminal. Experiencia en el área sanitaria 14 de la agencia valenciana de salud. Arch. Esp. Urol, 2007; 60: 273.

25. Cardona Maya W, Berdugo J, Cadavid Jaramillo A. The effects of male age on semen parameters: analysis of 1364 men attending an andrology center. Aging male; Epub ahead of print, 2009.

26. Sartorius G.A, Nieschlag E. Paternal age and reproduction. Hum. Reprod. Update; Epub ahead of print, 2009.

*27. Levitas E, Lunenfeld E, Weisz N, Friger M, Potashnik G. Relationship between age and semen parameters in men with norma sperm concentration: análisis of 6022 semen samples. Androl, 2007; 39: 45 . 
28. Schwartz D, Laplanche A, Jouannet P, David G. Within-subject variability of human semen in regard to sperm count, volume, total number of spermatozoa and length of abstinence." J. Reprod. Fertil, 1979; 57: 391.

29. Tash JA, McGovern JH, Schlegel PN. Acquired hypogonadotropic hypogonadism presenting as decreased seminal volume. Urol, 2000; 56: 669.

30. Wyrobek A. Methods and concepts in detecting abnormal reproductive outcomes of paternal origin. Reprod. Toxicol, 1993; 7: 3.

31. Wyrobek AJ. Methods for evaluating the effects of environmental chemicals on humen sperm production. Env. Health. Perspec, 1983; 48: 53.

*32. Mac Leod J. Human seminal cytology as a sensitive indicator of the germinal epithelium. Int. J. Fertil, 1964; 9: 281.

*33. Yanagimachi R. Mammalian fertilization. In: Knobil E, Neill JD (eds). The physiology of reproduction. P p 189-317, Raven Press, New York, 1994.

**34. Henkel R, Maass G, Schuppe H.C, Jung A, Schubert J, Schill WB. Molecular aspects of declining sperm motility in older men. Fertil. Steril, 2005; 84: 1430 .

35. Silverberg KM, Turner T. Evaluation of sperm. In: Gardner DK, Weissman A, Howles CM, Shoham $\mathrm{Z}$ (eds.). Textbook of assisted reproductive techniques. Laboratory and clinical perspectives. Pp 61-76, Taylor and Francis, London, 2001. 